\title{
Clinical and psychological outcomes of patients undergoing Retrograde Intrarenal Surgery and Miniaturised Percutaneous Nephrolithotomy for kidney stones. A preliminary study
}

\author{
Davide Di Mauro ${ }^{1}$, Valentina Lucia La Rosa ${ }^{2}$, Sebastiano Cimino ${ }^{1}$, Eugenio Di Grazia ${ }^{3}$ \\ ${ }^{1}$ Department of Urology, University of Catania, Catania, Italy; \\ ${ }^{2}$ Unit of Psychodiagnostics and Clinical Psychology, University of Catania, Catania, Italy; \\ ${ }^{3}$ Unit of Urology, Garibaldi Hospital, Catania, Italy
}

\begin{abstract}
Summary Purpose: To assess disease-specific and health-related QoL, anxiety and depression as well as satisfaction regarding retrograde intrarenal surgery (RIRS) and miniaturized percutaneous nephrolithotomy (mPCNL) intervention for kidney stones up to $2.5 \mathrm{~cm}$. Secondarily, pain as well as perioperative and postoperative patient outcomes were evaluated.

Methods: 60 consecutive patients with kidney stones of dimensions not exceeding $2.5 \mathrm{~cm}$ were enrolled in the study of which 30 underwent RIRS and $30 \mathrm{mPCNL}$. Perioperative characteristics (age, gender, body mass index (BMI), stone side and size, previous interventions for kidney stones and duration of hospitalization) and surgical outcomes (hemoglobin drop, stone-free rate, visual analogue scale (VAS), stenting time, size of ureteral access sheath (UAS) deployment, and postoperative complications) of patients were collected. Quality of life and psychological outcomes were evaluated using validated questionnaires.
\end{abstract}

Results: No significant differences were found between the two groups in terms of age, gender, BMI, stone side and size ( $p>$ 0.05 ). Significant differences between the mPCNL and the RIRS groups were found regarding stenting time $(p=0.032)$ and duration of hospital stay $(p<0.001)$. The stone-free rates of $M P C N L$ vs RIRS were not significantly different between the two groups $(73.3 \%$ vs $66.7 \%, p>0.05)$. Peri- and postoperative complications were not statistically different between the two groups $(p>0.05)$. RIRS group reported higher anxiety and depression scores compared with the mPCNL group (3 [range 0-15] vs 15 [range 6-24], $p<0.01)$. We found significant differences between the two groups in social $(p<0.05)$ and vitality $(p<0.01)$ scores. VAS pain score was significantly lower in the mPCNL group than in the RIRS one $(p<0.05)$. Conclusions: These results open new scenarios in the treatment of kidney stones up to $2.5 \mathrm{~cm}$ when RIRS and mPCNL have interchangeable indications. Since in our experience complications and success rate are similar, the surgical choice of switching from RIRS to $m P C N L$ in real-time and viceversa may be proposed to the patient in the preoperative counseling.

KEY WORDS: RIRS; mPCNL; Kidney stones; Quality of life; Satisfaction.

Submitted 24 September 2019; Accepted 24 October 2019

\section{INTRODUCTION}

Retrograde Intrarenal Surgery (RIRS) is recommended as the standard treatment for small to medium $(<2 \mathrm{~cm})$ renal stones by the European Association of Urology (EAU) Guidelines. The reason is that a high stone-free rate of usually more than $90 \%$ can be achieved, which is potentially related to fewer complications in comparison with percutaneous nephrolithotomy (PCNL). Many studies attempted to treat stones of a size up to $2.5 \mathrm{~cm}$ with RIRS in order to decrease potential PCNL morbidity. Contrariwise, RIRS failure rates range from $8 \%$ to $10 \%$ due to a difficult impassable ureter. Additionally, the failure rate to insert a standard ureteral access sheath (UAS) is probably higher because in most failed ureteroscopies even smaller ureteroscopes cannot be inserted. In these cases, a stent needs to be placed and the procedure postponed. After the ureteral relaxed, RIRS can be repeated. Double-staged procedures are frustrating for urologists and for patients. Moreover, they increase significantly costs related to additional stone treatments and prolonged hospital stay. A real-time surgical alternative, as morbid and mini-invasive as RIRS, is needed to avoid staged-procedures. In the last few years many studies reconsidered miniaturized PCNL (mPCNL) as an alternative, which is a more effective approach compared with RIRS reporting a better stone-free rate and a similar complications rate (1).

Furthermore, patients often undergo for several days postoperative ureteral stenting to prevent from complications related to residual fragments in the ureteral passage, potential ureteral edema or post UAS inflammation. Postoperative ureteral stenting has impacts on the quality of life (QoL) for patients, a fact often not considered in RIRS and mPCNL researches. The principles of evidencebased medicine recommend considering both clinicalreported outcome (CRO) parameters and patient-reported outcomes (PROs) (2). Despite the adoption of mPCNL and RIRS techniques into clinical practice, comparative clinical data, assessing patient satisfaction, taking perioperative and postoperative morbidities into account, is lacking.

The primary aim of our study was to assess disease-specific and health-related QoL, anxiety and depression as well as satisfaction regarding RIRS and mPCNL intervention for kidney stones up to $2.5 \mathrm{~cm}$. Secondarily, pain as well as perioperative and postoperative patient outcomes were evaluated.

No conflict of interest declared. 


\section{MATERIALS AND METHODS}

\section{Study design and ethical approval}

The study conducted at a tertiary hospital and was carried out in accordance with the Declaration of Helsinki and the STROBE guidelines. Informed consent was obtained from all participants before the study.

\section{Study variables}

Perioperative characteristics (age, gender, body mass index (BMI), stone side and size, previous interventions for kidney stones and duration of hospitalization) and surgical outcomes (hemoglobin drop, stone-free rate (SFR), visual analogue scale (VAS), stenting time, size of UAS deployment, and postoperative complications) of patients treated between January 2018 and January 2019 were collected.

All pre-operative imaging was accomplished by computed tomography (CT) scans with $3 \mathrm{~mm}$ cuts. Stone size and volumes were collated using the EAU stone volume formula (volume $=\pi / 6 \AA \sim$ Length $\mathrm{x}$ Width $\mathrm{x}$ Depth) using CT scan measurements. Stone-free rate was calculated taking stone fragments less than $4 \mathrm{~mm}$ by KUB radiographic or sonographic evaluation after 3 months postsurgery into account.

Health Related Quality of Life (HRQoL) was evaluated using the Italian adaptation of the Wisconsin Stone Quality of Life questionnaire (WISQOL)3. It is a 28-item, selfadministered instrument designed to evaluate the effect of kidney stones on patients' QoL. Through a 5-point Likert scale, it is possible to evaluate the following domains: activity/energy level, sleep patterns, social functioning, therapy compliance, physical symptoms, family life, intimacy, and emotional health. A higher score is associated to better QoL (3).

Anxiety and depression levels were assessed through the Hospital Anxiety and Depression Scale (HADS) (4). It is a self-report questionnaire designed to detect clinical cases of depression and anxiety. It includes two subscales evaluating anxiety (HADS-A) and depression (HADS-D), respectively. Each subscale consists of 7 items to be rated on a four-point scale (0-3). The subscales have a maximum score of 21 and a score above 11 is considered clinically significant (4).

Both the questionnaires were administered 6 months after surgery. Furthermore, we assessd levels of perceived pain and satisfaction regarding the treatment using a VAS 10-point scale (5). Finally, the procedures were compared regarding surgical outcomes and stonefree rate. Peri- and postoperative complications of procedures were classified using the modified Clavien-Dindo classification for PCNL surgery (6).

\section{Participants}

Assuming two balanced groups, a sample size of 58 patients achieve $80 \%$ of power with a significance level of $5 \%$ to detect a minimum mean difference of 0.78 .60 patients with kidney stones of dimensions not exceeding $2.5 \mathrm{~cm}$ were enrolled in the study of which 30 underwent RIRS and $30 \mathrm{mPCNL}$.

Exclusion criteria were the age under 18 years, transplantation or urinary derivation, congenital anomalies, renal cancer, pregnancy, cardiovascular or pulmonary comorbidities, coagulation disorders only for PCNL surgery, psychological and/or psychiatric diseases, cognitive and linguistic abilities not sufficient to understand and interpret questionnaires used in the study.

\section{Surgical techniques}

mPCNL and RIRS were performed under general anesthesia and antibiotic prophylaxis with Gentamicine 80 $\mathrm{mg}$ when preoperative urine culture was negative. Antibiotic therapy was performed according to antibiotic sensibility when urine culture was positive at least 3 days before surgery and continued 3 days afterwards.

\section{$m P C N L$}

A transurethral $5 \mathrm{~F}$ open-ended catheter was positioned in the ureter to inject contrast dye in the upper tract in a Galdakao-modified supine Valdivia position (7).

Puncture of the calyceal system was performed under both ultrasound and fluoroscopy guidance. After placing a hydrophilic safety guide wire (Bard Nicore nitinol guidewire with hydrophilic coating. 0,035" x $150 \mathrm{~cm}$ stiff shaft-straight tip), a $10 \mathrm{~F}$ dual-lumen angiography catheter (Boston Scientific) and a second safety guide wire were inserted (Sensor TM - PTFE- Nitinol Guidewire with hydrophilic tip - Boston Scientific). One-shot tract dilation was accomplished over the Sensor ${ }^{\mathrm{TM}}$ guidewire with metal dilatator (Karl Storz) and a 15 or $17.5 \mathrm{Fr}$ Amplatz sheath (Karl Storz) was placed. A 12 F Nephroscope (Karl Storz) was inserted through the Amplatz sheath to fragment kidney stones using dusting settings with a 500 $\mu \mathrm{m}$ fiber $(0.3 \mathrm{~J} \times 20-30 \mathrm{~Hz})$. Residual fragments were washed out with a vacuum cleaner effect. The procedure was finished with the placement of an $8 \mathrm{~F}$ nephrostomy (Flexima ${ }^{T M}$-Boston Scientific) or a double J stent (BardInlay ureteral stent 6 F 24-26-28) with attached strings coming out the urethra (when tubeless mPCNL was performed). The nephrostomy was removed after confirming a free ureter passage by antegrade ureterography (24$36 \mathrm{~h}$ after the procedure). In tubeless mPCNL, the ureteral stent was removed 3 to 7 days after the intervention pulling on the attached strings.

\section{RIRS}

RIRS was started with cystoscopic (Rigid cystoscope $19 \mathrm{~F}$ Karl Storz) insertion of a safety guide-wire (Bard Nicore nitinol guidewire with hydrophilic coating, 0,035" x 150 $\mathrm{cm}$ stiff shaft-straight tip) in supine lithotomic position. A 7 F semirigid ureteroscope (Karl Storz) was used to exclude intraureteral lesions, stones or insufficient ureter dilation. A second guide wire was inserted, and a 10/12 Fr UAS, (Retrace ${ }^{\circledR}$ Coloplast) was deployed over the hydrophilic guidewire inside the ureter if ureteral walls compliance permitted under fluoroscopic guidance. A $7.5 \mathrm{~F}$ flexible fiberoptic ureteroscope (Flex $X 2^{\circledR}$ - Karl Storz) was used for the complete inspection of the pelvicaliceal system. Stones were fragmented using a Holmium laser dusting set with a $200 \mu \mathrm{m}$ fiber (Lumenis Pulse ${ }^{T M} 120 H$ ). Some removal of fragments was not systematically performed using a nitinol basket (Zero-Tip ${ }^{T M}$ Boston Scientific) and postoperative stenting (7-14 days) (Bard Inlay ureteral stent $6 \mathrm{~F}$ 24-26-28), with an 
attached string were used (to be extracted by pulling on the string).

\section{Statistical analysis}

Statistical analyses were performed using $\mathrm{R}$ package "Rcmdr" (version 2.5-1). A p value $<0.05$ was considered statistically significant.

Quantitative variables were expressed as mean \pm standard deviation (SD) or as median (interquartile range: IQR), when appropriate. Categorical variables were expressed as frequencies (percentages).

After testing the non-normality of the distribution of quantitative variables with the Shapiro Wilk test, nonparametric tests were used for data analysis.

Chi-squared test was utilized to compare categorical variables and Mann-Whitney test to underline possible significant differences between mPCNL and RIRS for the clinical and psychological outcomes examined in this study.

\section{RESULTS}

A total of 30 patients who underwent mPCNL and 30 patients who underwent RIRS were enrolled in this study. The mean age of the patients was 55 (range 4459) years in the mPCNL group and 57.50 (range 47-61) years in the RIRS group. No significant differences were found between the two groups in terms of age, gender, BMI, stone side and size ( $p>0.05)$. However, the two groups were significantly different in terms of previous interventions for kidney stones $(p=0.002)$. All the demographic data and stone characteristics of the two groups are summarized in Table 1.

As shown in Table 2, significant differences between the $\mathrm{mPCNL}$ and the RIRS groups were found regarding stenting time ( $\mathrm{p}=0.032)$ and duration of hospital stay ( $\mathrm{p}$ $<0.001$ ). The stone-free rates of mPCNL vs RIRS were not significantly different between the two groups (73.3\% vs $66.7 \%, \mathrm{p}>0.05)$. Peri- and postoperative complications were not statistically different between the two groups ( $p>0.05$ ). We found a significant difference regarding hemoglobin drop values in favor of RIRS, even if no patient needed blood transfusions $(p<0.001)$.

Table 1.

Demographic data and stone characteristics of the sample.

\begin{tabular}{|c|c|c|c|c|}
\hline & & $\begin{array}{c}\text { mPCNL group } \\
(n=30)\end{array}$ & $\begin{array}{l}\text { RIRS group } \\
(n=30)\end{array}$ & $P$ value \\
\hline \multirow[t]{2}{*}{ Gender } & Women & $22(73.3)$ & $14(46.7)$ & 0.064 \\
\hline & Men & $8(26.7)$ & $16(53.3)$ & \\
\hline Age (yr) & & $55.00[44.00,59.00]$ & $57.50[47.00,61.00]$ & 0.415 \\
\hline $\mathrm{BMI}\left(\mathrm{kg} / \mathrm{m}^{2}\right)$ & & $26.48[24.02,27.54]$ & $25.43[23.72,32.33]$ & 0.750 \\
\hline \multirow[t]{5}{*}{ Previous interventions } & None & $11(36.7)$ & $21(70.0)$ & $0.002^{* *}$ \\
\hline & ESWL & $2(6.7)$ & $4(13.3)$ & \\
\hline & PCNL & $7(23.3)$ & $0(0.0)$ & \\
\hline & RIRS & $10(33.3)$ & $4(13.3)$ & \\
\hline & URS & $0(0.0)$ & $1(3.3)$ & \\
\hline \multirow[t]{3}{*}{ Stone side } & Bilateral & $2(6.7)$ & $3(10.0)$ & 0.737 \\
\hline & Right & $16(53.3)$ & $13(43.3)$ & \\
\hline & Left & $12(40.0)$ & $14(46.7)$ & \\
\hline Stone size $(\mathrm{cm})$ & & $2.00[1.62,2.50]$ & $1.55[1.20,2.50]$ & 0.219 \\
\hline
\end{tabular}

Table 2.

Postoperative parameters in study groups.

\begin{tabular}{|lcccc|}
\hline & $\begin{array}{c}\text { mPCNL group } \\
(\mathbf{n}=\mathbf{3 0 )}\end{array}$ & $\begin{array}{c}\text { RIRS group } \\
(\mathbf{n}=\mathbf{3 0 )}\end{array}$ & P value \\
\hline Clavien complications (\%) & Grade 0 & $28(93.3)$ & $28(93.3)$ & 1.000 \\
& Grade I & $2(6.7)$ & $2(6.7)$ & \\
\hline Stone-free rate (\%) & $<4 \mathrm{~mm}$ & $22(73.3)$ & $20(66.7)$ & 0.779 \\
& $>4 \mathrm{~mm}$ & $8(26.7)$ & $10(33.3)$ & \\
\hline Hospital stay (d) & $6.00[4.00,9.00]$ & $2.00[1.00,5.00]$ & $<0.001$ \\
\hline Stenting time (d) & $7.00[0.00,10.00]$ & $8.00[5.00,14.75]$ & $0.032^{*}$ \\
\hline Hemoglobin (Hb) drop (mg/dL) & $1.15[0.9,2.50]$ & $0.5[0.00,0.7]$ & $<0.001$ \\
\hline Data are expressed as median [rangé and as frequencies (percentages). ${ }^{*} p<.05$. & \\
\hline
\end{tabular}

\section{Table 3.}

Psychological outcomes in study groups.

\begin{tabular}{|c|c|c|c|}
\hline & $\begin{array}{c}\text { mPCNL group } \\
(n=30)\end{array}$ & $\begin{array}{l}\text { RIRS group } \\
(n=30)\end{array}$ & $P$ value \\
\hline$\overline{\text { HADS (anxiety) }}$ & $3.00[0.00,7.00]$ & $7.00[4.00,14.00]$ & $0.006^{* *}$ \\
\hline HADS (depression) & $0.00[0.00,5.00]$ & $8.00[1.00,10.75]$ & $<0.001 * *$ \\
\hline HADS (total) & $3.00[0.00,15.00]$ & $15.00[6.00,24.00]$ & $0.001^{* *}$ \\
\hline QoL disease score & $87.50[50.00,95.33]$ & $65.60[45.35,85.15]$ & 0.179 \\
\hline QoL emotional score & $82.10[57.10,96.40]$ & $78.60[57.10,92.90]$ & 0.629 \\
\hline QoL social score & $100.00[81.30,100.00]$ & $84.40[63.30,96.90]$ & $0.028^{*}$ \\
\hline QOL vitality score & $100.00[66.65,100.00]$ & $50.00[33.30,91.70]$ & $0.004^{* *}$ \\
\hline QoL total score & $90.20[67.00,95.50]$ & $68.75[56.30,90.43]$ & 0.166 \\
\hline Satisfaction score & $10.00[9.00,10.00]$ & $10.00[7.00,10.00]$ & 0.098 \\
\hline VAS score & $2.00[1.00,5.75]$ & $5.00[4.00,7.00]$ & $0.032 *$ \\
\hline \multicolumn{4}{|c|}{$\begin{array}{l}\text { Data are expressed as median [rangej. } \\
\text { HADS: Hospital Anxiety and Depression Scale; QoL: Quality of Life; VAS: Visual Analogue Scale. } \\
{ }^{*} p<.05 ;{ }^{* *} p<.01 \text {. }\end{array}$} \\
\hline
\end{tabular}

Table 3 describes psychological and QoL outcomes assessed in the two groups. Statistically significant differences between the MPCNL and the RIRS groups were found in terms of HADS scores reporting higher anxiety and depression scores, compared with the mPCNL group (3 [range 0-15] vs 15 [range 6-24], $\mathrm{p}<0.01$ ).

Regarding QoL evaluated with the WISQOL questionnaire, we found significant differences between the two groups in social $(p<0.05)$ and vitality $(p<0.01)$ scores. No significant differences were found between the two groups in terms of satisfaction score ( $p>0.05$ ). VAS pain score was significantly lower in the mPCNL group than in the RIRS one $(p<0.05)$.

\section{Discussion}

Endourologic stone management comprises several minimally invasive techniques (PCNL, mPCNL, and RIRS) and takes several factors such as success rate, complication rate, comorbidities and technological facilities into account. Percutaneous surgery was developed in 1980 using at first large access sheaths (28-30 F) facilitating irrigation, debris drainage and active removing of large stones (8). In the last decade, mPCNL has gained attention because it involves a miniaturized nephroscope and offers a nephrostomy tract size $<20 \mathrm{~F}$ with the aim of decreasing complications associated with tract size during conventional PCNL while providing comparable SFR $(9,10)$. Besides, in the last two decades an increasing interest for RIRS in the management of kidney stones up to $2 \mathrm{~cm}$ with successful treatment of larger stones with fewer complications com- 
pared to standard PCNL using single or staged ureteroscopic procedures has been reported 6. Despite the evolution of MPCNL and RIRS techniques into clinical practice, there is still a lack of comparative clinical data assessing patient satisfaction taking into account perioperative and postoperative morbidity. The principles of evidence-based medicine (EBM) call for the consideration of both clinicalreported outcome (CRO) parameters and patient-reported outcomes (PROs) and the present analysis was conducted with the aim of identifying and critically comparing the outcomes of mPCNL with those of RIRS in the management of kidney stones up to $2,5 \mathrm{~cm}$, focusing both on clinical and psychological outcomes.

In our sample, stone-free rate was similar in mPCNL compared to the RIRS group $(73.3 \%$ vs $66.7 \%$, p > 0.05). Likely, mPCNL would reach the success rate more precociously than RIRS as most of RIRS performers use laser lithotripsy between 5 and $15 \mathrm{~W}$, using a dusting technique to reduce fragments to easily passible sandlike pieces. Instead, mPCNL may guarantee intraoperative stone clearance either by stone dusting or by fragments washed-out through Amplatz sheath (11). Validated Clavien-Dindo evaluation for complications did not reveal any statistically significant difference, while hospital stay was in favor for RIRS, as expected. In our experience, the length of stay is longer for the nephrostomy management in patients subjected to $\mathrm{MPCNL}$, as patients remain hospitalized while the nephrostomy is still inserted. Instead, in tubeless or a totally tubeless patients hospitalization times are overlapping with those of the RIRS. Patients subjected to RIRS are discharged precociously even if they carry a stent because they do not usually require any specific support until stent removal. Stenting time was significantly different between the MPCNL and the RIRS group (7 days [range 4-5] vs 8 days [range 514.75], $\mathrm{p}=0.032$ ). Prolonged stenting time in subjects undergoing RIRS is required to eliminate fragments and dust after surgery, which may take an indefinite time - an aspect of ongoing debate. A complete fragment clearance with a basket is very time consuming and requires an UAS of significant diameter to obtain an intraoperative "stone-free" status. On the other hand, RIRS failure rates due to a difficult impassable ureter range from $8 \%$ to $10 \%$ and the failure rate to insert a standard UAS is even higher because, in most failed ureteroscopy cases even the smaller diameter ureteroscopes cannot be insert. In these cases, stent placement is necessary, which postpones the procedure. After ureteral relaxation, RIRS can be repeated. The double-staged procedures may be frustrating either for Urologists or for patients and increase costs management. Differently, in the mPCNL fragment clearance is easily obtainable during surgery, allowing to decide whether to use either a stent or nephrostomy for a few days as a precaution to prevent complications (tubeless mPCNL) or not to use tubes at all (totally tubeless). Regarding psychological outcomes, we found significant differences between the two groups regarding QoL domains of social functioning $(\mathrm{p}<0.05)$ and vitality $(p<0.01)$. Furthermore, the RIRS group showed elevated anxiety and depression scores. Correlated to this data, also VAS pain scores were significantly lower in the mPCNL group compared with the RIRS group $(\mathrm{p}<$
0.05). These data are probably due also to the discomfort related to the postoperative stenting time which was longer in patients undergoing RIRS. Indeed, it has been demonstrated that stents provoke irritating voiding symptoms, hematuria, stent incrustation and fragmentation, back pain, stent migration, infection, pyelonephritis, and ureteral trauma $(12,13)$. These complications may significantly affect patients QoL and could explain the data in favor of mPCNL regarding psychological outcomes (14). Even though several studies demonstrated a significant difference in terms of discomfort and distress of stented and not stented patients, deeper insights are needed. Ringel et al. reported that $32.7 \%$ of their patients had ureteral stents removed because of complications (15). These observations open new scenarios in the treatment of urinary stones for which RIRS and mPCNL have interchangeable indications. Since the complications and the success rate are similar, the surgical choice of switching from RIRS to mPCNL and viceversa may be proposed to patients during pre-operative counseling. Moreover, supine position modified according to Galdakao variant allows in the selected cases of kidney stones up to 2.5 $\mathrm{cm}$, an easy, no time-consuming switch from retrograde to anterograde treatment and vice-versa when patients are unfit for one over the other technique. This could potentially prevent a double-staged procedure, not compliant ureter, UAS insertion failures, impacted stones, anatomical anomalies, inadequate laser dusting for stone physical characteristics and dilated upper tract system. Our study, if corroborated by others on this topic, introduces a new possible paradigm in the treatment of urinary stones up to $2.5 \mathrm{~cm}$, for which the technique (RIRS or $\mathrm{mPCNL}$ ) to be used is not decided in the preoperative setting, but in progress during the surgery.

\section{Strengths and limitations}

Our study represents an important contribution to the comparison between the surgical procedures most used in the treatment of kidney stones, not only in reference to clinical and surgical outcomes but also to psychological and QoL ones. For this reason, it may be a reference with regard to the clinical and multidisciplinary management of these patients.

However, this study has some limitations. First of all, the sample is small, and it is therefore necessary to conduct further studies on larger samples to confirm the results of the study. Furthermore, some data are based on selfreported questionnaires with consequent risk of bias linked to the patient's social desirability. Finally, we did not include a specific questionnaire to assess patients experience of stenting. Further studies are needed about this topic in order to better understand the real impact of stenting on QoL and psychological wellbeing.

\section{Conclusions}

RIRS is usually considered less invasive than mPCNL and preferred in stones up to $2-2.5 \mathrm{~cm}$ but in several cases planned RIRS is not feasible because of unpredictable difficulties forcing the surgeon to place a ureteral stent or nephrostomy and postpone the surgery.

In our study, we reported similar outcomes in terms of 
success-rate, complications and quality of life in up to $2.5 \mathrm{~cm}$ stones comparable groups undergoing RIRS and mPCNL. Interestingly, the increased stenting time in patients subjected to RIRS compared to those subjected to mPCNL significantly impacted on QoL. These observations open new scenarios in the treatment of kidney stones when RIRS and mPCNL have interchangeable indications. Since in our experience complications and success rate are similar, the surgical choice of switching from RIRS to mPCNL in real-time and viceversa may be proposed to the patient in the preoperative counseling.

\section{REFERENCES}

1. Davis NF, Quinlan MR, Poyet C, et al. Miniaturised percutaneous nephrolithotomy versus flexible ureteropyeloscopy: a systematic review and meta-analysis comparing clinical efficacy and safety profile. World J Urol. 2018; 36:1127-38.

2. Garcia SF, Cella D, Clauser SB, et al. Standardizing patientreported outcomes assessment in cancer clinical trials: a patientreported outcomes measurement information system initiative. J Clin Oncol. 2007; 25:5106-12.

3. Penniston KL, Antonelli JA, Viprakasit DP, et al. Validation and Reliability of the Wisconsin Stone Quality of Life Questionnaire. J Urol. 2017; 197:1280-8.

4. Zigmond AS, Snaith RP. The hospital anxiety and depression scale. Acta Psychiatr Scand. 1983; 67:361-70.

5. Heller GZ, Manuguerra M, Chow R. How to analyze the Visual Analogue Scale: Myths, truths and clinical relevance. Scand J Pain. 2016; 13:67-75
6. de la Rosette JJ, Opondo D, Daels FP, et al. Categorisation of complications and validation of the Clavien score for percutaneous nephrolithotomy. Eur Urol. 2012; 62:246-55

7. Scoffone CM, Cracco CM, Cossu M, et al. Endoscopic combined intrarenal surgery in Galdakao-modified supine Valdivia position: a new standard for percutaneous nephrolithotomy? Eur Urol. 2008; 54:1393-403.

8. Hyams ES, Munver R, Bird VG, et al. Flexible ureterorenoscopy and holmium laser lithotripsy for the management of renal stone burdens that measure 2 to $3 \mathrm{~cm}$ : a multi-institutional experience. $J$ Endourol. 2010; 24:1583-8.

9. Mariani AJ. Combined electrohydraulic and holmium:YAG laser ureteroscopic nephrolithotripsy of large (greater than $4 \mathrm{~cm}$ ) renal calculi. J Urol. 2007; 177:168-73; discussion73.

10. Monga M, Oglevie S. Minipercutaneous nephrolithotomy. J Endourol. 2000; 14:419-21.

11. Jackman SV, Docimo SG, Cadeddu JA, Bishoff JT, Kavoussi LR, Jarrett TW. The "mini-perc" technique: a less invasive alternative to percutaneous nephrolithotomy. World J Urol. 1998; 16:371-4.

12. De S, Autorino R, Kim FJ, et al. Percutaneous nephrolithotomy versus retrograde intrarenal surgery: a systematic review and metaanalysis. Eur Urol. 2015; 67:125-37.

13. Ucuzal M, Serce P. Ureteral Stents: Impact on Quality of Life. Holist Nurs Pract. 2017; 31:126-32.

14. Kelly T, Kelly MH. Living with ureteric stents: a phenomenological study. Br J Nurs. 2019; 28:S29-S37.

15. Ringel A, Richter S, Shalev M, Nissenkorn I. Late complications of ureteral stents. Eur Urol. 2000; 38:41-4.

\section{Correspondence}

Davide Di Mauro, MD

davidedimauro84@virgilio.it

Sebastiano Cimino, MD

Department of Urology, University of Catania, Catania (Italy)

Valentina Lucia La Rosa, Psy. D.

psicolarosa@gmail.com

Unit of Psychodiagnostics and Clinical Psychology, University of Catania, Catania (Italy)

Eugenio Di Grazia, MD (Corresponding Author)

Unit of Urology, Garibaldi Hospital, Catania (Italy) 